\title{
Are we exposed to halogenated flame retardants from both primary and secondary sources?
}

Congqiao Yang ${ }^{1 *}$, Simona Rozarka Jílková ${ }^{2 *}$, Lisa Melymuk $^{2 \S}$, Shelley Anne Harris ${ }^{3,4}$, Liisa M. Jantunen ${ }^{5,1}$, Jessica Pertili ${ }^{6}$, Louise Winn ${ }^{6}$, Miriam L. Diamond $^{1,3 \S}$

${ }^{1}$ Department of Earth Sciences, University of Toronto, Toronto, Ontario, Canada M5S 3B1

${ }^{2}$ RECETOX, Masaryk University, 62500 Brno, Czech Republic

${ }^{3}$ Dalla Lana School of Public Health, University of Toronto, Toronto, Ontario, Canada M5T 3M7

${ }^{4}$ Occupational Cancer Research Centre, Cancer Care Ontario, Toronto, Ontario, Canada M5G $1 \mathrm{X} 3$

${ }^{5}$ Air Quality Research Processes Section, Environment and Climate Change Canada, Egbert, Ontario, Canada LOL 1N0

${ }^{6}$ School of Environmental Studies, Queen's University, Kingston, Ontario, Canada K7L 3N6

* Co-first authors

${ }^{\S}$ Co-corresponding authors

$\underline{\text { miriam.diamond@utoronto.ca }}$

lisa.melymuk@ recetox.muni.cz 
Table S1: List of NFRs and PBDEs for analysis, along with CAS numbers, molecular formula, molecular weight, and $\log K_{\mathrm{OW}}$ and $\log \mathrm{K}_{\mathrm{OA}}{ }^{1,2}$

\begin{tabular}{|c|c|c|c|c|c|}
\hline & Full Name & $\begin{array}{l}\text { CAS, Molecular } \\
\text { Formula }\end{array}$ & $\begin{array}{l}\text { Molecular } \\
\text { weight } \\
(\mathrm{g} / \mathrm{mol})\end{array}$ & LogKow & LogKoA \\
\hline \multicolumn{6}{|c|}{ Novel flame-retardants (NFRs) } \\
\hline TBP-AE & $\begin{array}{l}\text { Allyl 2,4,6-tribromophenyl ether } \\
\text { Also known as: } \\
\text { 1,3,5-Tribromo-2-(prop-2-en-1- } \\
\text { yloxy)benzene }\end{array}$ & $3278-89-5, \mathrm{C}_{9} \mathrm{H}_{7} \mathrm{Br}_{3} \mathrm{O}$ & 371 & 5.6 & 8.6 \\
\hline PBBz & Pentabromobenzene & $608-90-2, \mathrm{C}_{6} \mathrm{HBr}_{5}$ & 473 & 6.4 & 9.1 \\
\hline PBT & Pentabromotoluene & 87-83-2, $\mathrm{C}_{7} \mathrm{H}_{3} \mathrm{Br}_{5}$ & 487 & 7.0 & 9.6 \\
\hline PBEB & Pentabromoethyl benzene & $85-22-3, \mathrm{C}_{8} \mathrm{H}_{5} \mathrm{Br}_{5}$ & 501 & 7.5 & 10 \\
\hline HBB & Hexabromobenzene & $87-82-1, \mathrm{C}_{6} \mathrm{Br}_{6}$ & 552 & 7.3 & 10.4 \\
\hline EH-TBB & Ethylhexyl-tetrabromobenzoate & \begin{tabular}{|l}
$26040-51-7$ \\
$\mathrm{C}_{15} \mathrm{H}_{19} \mathrm{Br}_{4} \mathrm{O}_{2}$ \\
\end{tabular} & 550 & 8.8 & 12.3 \\
\hline BEH-TEBP & Bis(2-ethylhexyl) tetrabromophthalate & \begin{tabular}{|l}
$26040-51-7$ \\
$\mathrm{C}_{24} \mathrm{H}_{34} \mathrm{Br}_{4} \mathrm{O}_{4}$ \\
\end{tabular} & 706 & 12 & 16.9 \\
\hline s-DDC-CO & Dechlorane Plus (syn and anti isomers) & $13560-89-9, \mathrm{C}_{18} \mathrm{H}_{12} \mathrm{Cl}_{12}$ & 654 & 11.3 & 14.8 \\
\hline a-DDC-CO & Dechlorane Plus (syn and anti isomers) & $13560-89-9, \mathrm{C}_{18} \mathrm{H}_{12} \mathrm{Cl}_{12}$ & 654 & 11.3 & 14.8 \\
\hline OBTMPI & Octabromotrimethylphenyl indane & $1084889-51-9, \mathrm{C}_{18} \mathrm{H}_{12} \mathrm{Br}_{8}$ & 868 & 13 & 17.8 \\
\hline DBDPE & Decabromodiphenylethane & $84852-53-9, \mathrm{C}_{14} \mathrm{H}_{4} \mathrm{Br}_{10}$ & 971 & 13.6 & 19.2 \\
\hline \multicolumn{6}{|c|}{ Polybrominated diphenyl ethers (PBDEs) } \\
\hline BDE-17 & 2,2',4-Tribromodiphenyl ether & \begin{tabular}{|l|}
$147217-75-2$ \\
$\mathrm{C}_{13} \mathrm{H}_{9} \mathrm{Br}_{3} \mathrm{O}_{2}$ \\
\end{tabular} & 437 & 5.88 & 9.3 \\
\hline BDE-28 & 2,4,4'-Tribromodiphenyl ether & $41318-75-6, \mathrm{C}_{12} \mathrm{H}_{7} \mathrm{Br}_{3} \mathrm{O}$ & 407 & 5.88 & 9.5 \\
\hline BDE-47 & 2,2',4,4'-Tetrabromodiphenyl ether & $5436-43-1, \mathrm{C}_{12} \mathrm{H}_{6} \mathrm{Br}_{4} \mathrm{O}$ & 486 & 6.77 & 10.5 \\
\hline BDE-49 & 2,2',4,5'-Tetrabromodiphenyl ether & $243982-82-3, \mathrm{C}_{12} \mathrm{H}_{6} \mathrm{Br}_{4} \mathrm{O}$ & 486 & $6.37^{*}$ & - \\
\hline BDE-66 & 2,3',4,4'-Tetrabromodiphenyl ether & $189084-61-5, \mathrm{C}_{12} \mathrm{H}_{6} \mathrm{Br}_{4} \mathrm{O}$ & 486 & 6.77 & 10.8 \\
\hline BDE-71 & 2,3',4',6-Tetrabromodiphenyl ether & $189084-62-6, \mathrm{C}_{12} \mathrm{H}_{6} \mathrm{Br}_{4} \mathrm{O}$ & 486 & 6.77 & 10.4 \\
\hline BDE-85 & 2,2',3,4,4'-Pentabromodiphenyl ether & $182346-21-0, \mathrm{C}_{12} \mathrm{H}_{5} \mathrm{Br}{ }_{5} \mathrm{O}$ & 565 & 7.66 & 11.7 \\
\hline BDE-99 & 2,2',4,4',5-Pentabromodiphenyl ether & $60348-60-9, \mathrm{C}_{12} \mathrm{H}_{5} \mathrm{Br}_{5} \mathrm{O}$ & 565 & 7.66 & 11.3 \\
\hline BDE-100 & 2,2',4,4',6-Pentabromodiphenyl ether & 189084-64-8, $\mathrm{C}_{12} \mathrm{H}_{5} \mathrm{Br}_{5} \mathrm{O}$ & 565 & 7.66 & 11.1 \\
\hline BDE-138 & 2,2',3,4,4',5'-Hexabromodiphenyl ether & $182677-30-1, \mathrm{C}_{12} \mathrm{H}_{4} \mathrm{Br}_{6} \mathrm{O}$ & 644 & 8.55 & - \\
\hline BDE-153 & $2,2^{\prime}, 4,4$ ',5,5'-Hexabromodiphenyl ether & $68631-49-2, \mathrm{C}_{12} \mathrm{H}_{4} \mathrm{Br}_{6} \mathrm{O}$ & 644 & 8.55 & 11.8 \\
\hline BDE-154 & $2,2^{\prime}, 4,4$ ',5,6'-Hexabromodiphenyl ether & $207122-15-4, \mathrm{C}_{12} \mathrm{H}_{4} \mathrm{Br}_{6} \mathrm{O}$ & 644 & 8.55 & 11.9 \\
\hline BDE-183 & $\begin{array}{l}\text { 2,2',3,4,4',5',6-Heptabromodiphenyl } \\
\text { ether }\end{array}$ & $207122-16-5, \mathrm{C}_{12} \mathrm{H}_{3} \mathrm{Br} 7 \mathrm{O}$ & 722 & 9.44 & 12 \\
\hline BDE-190 & $\begin{array}{l}2,2^{\prime}, 3^{\prime}, 4,4^{\prime}, 5^{\prime}, 6-\text { Heptabromodiphenyl } \\
\text { ether }\end{array}$ & $189084-68-2, \mathrm{C}_{12} \mathrm{H}_{3} \mathrm{Br}_{7} \mathrm{O}$ & 722 & 9.44 & - \\
\hline BDE-209 & Decabromodiphenyl ether & $1163-19-5, \mathrm{C}_{12} \mathrm{Br}_{10} \mathrm{O}$ & 959 & 12.1 & - \\
\hline
\end{tabular}

* calculated in Virtual Computational Chemistry Laboratory (http://www.vcclab.org)

-: not available 
Table S2: Method detection limits (MDL) for sample types. MDL for cell phones (wiped area $=50 \mathrm{~cm}^{2}$ ) is 2 times of that for other electronic products (wiped area $=100 \mathrm{~cm}^{2}$ ).

\begin{tabular}{|c|c|c|c|c|c|}
\hline & & $\begin{array}{c}\text { Air } \\
\left(\mathrm{pg} / \mathrm{m}^{3}\right)\end{array}$ & $\begin{array}{l}\text { Dust } \\
\text { (ng/g) }\end{array}$ & $\begin{array}{l}\text { Product Wipes } \\
\qquad\left(\mathrm{pg} / \mathrm{cm}^{2}\right)\end{array}$ & $\begin{array}{c}\text { Hand Wipes } \\
\left(\mathrm{pg} / \mathrm{cm}^{2}\right)\end{array}$ \\
\hline \multirow{11}{*}{ NFRs } & TBP-AE & 0.14 & 0.10 & 0.08 & 0.03 \\
\hline & PBBz & 0.03 & 0.03 & 0.02 & 0.01 \\
\hline & PBT & 0.07 & 0.05 & 0.05 & 0.02 \\
\hline & PBEB & 0.07 & 0.05 & 0.04 & 0.02 \\
\hline & HBB & 0.03 & 0.02 & 0.02 & 0.01 \\
\hline & EH-TBB & 1.4 & 1.0 & 0.8 & 0.30 \\
\hline & ВЕН-ТEВP & 3.5 & 2.5 & 2.0 & 0.80 \\
\hline & s-DDC-CO & 0.34 & 0.25 & 0.2 & 0.08 \\
\hline & a-DDC-CO & 0.34 & 0.25 & 0.2 & 0.08 \\
\hline & OBTMPI & 7.0 & 5.0 & 4.0 & 1.6 \\
\hline & DBDPE & 8.4 & 6.0 & 4.8 & 2.0 \\
\hline \multirow{15}{*}{ PBDEs } & BDE-17 & 0.22 & 0.15 & 0.12 & 0.05 \\
\hline & BDE-28 & 0.22 & 0.15 & 0.12 & 0.05 \\
\hline & BDE-47 & 0.22 & 0.15 & 0.12 & 0.05 \\
\hline & BDE-49 & 0.22 & 0.15 & 0.12 & 0.05 \\
\hline & BDE-66 & 0.22 & 0.15 & 0.12 & 0.05 \\
\hline & BDE-71 & 0.22 & 0.15 & 0.12 & 0.05 \\
\hline & BDE-85 & 0.22 & 0.15 & 0.12 & 0.05 \\
\hline & BDE-99 & 0.22 & 0.15 & 0.12 & 0.05 \\
\hline & BDE-100 & 0.22 & 0.15 & 0.12 & 0.05 \\
\hline & BDE-138 & 0.29 & 0.20 & 0.16 & 0.06 \\
\hline & BDE-153 & 0.29 & 0.15 & 0.16 & 0.06 \\
\hline & BDE-154 & 0.22 & 0.15 & 0.12 & 0.05 \\
\hline & BDE-183 & 0.29 & 0.20 & 0.16 & 0.06 \\
\hline & BDE-190 & 0.14 & 0.10 & 0.08 & 0.03 \\
\hline & BDE-209 & 18 & 13 & 10 & 4.5 \\
\hline
\end{tabular}


Table S3: Analysis of SRM 2585 house dust samples (ng/g; $n=5)$ for NFRs and PBDEs.

\begin{tabular}{|c|c|c|c|c|}
\hline & & $\begin{array}{l}\text { SRM measurements } \\
\qquad(\mathrm{n}=5)\end{array}$ & $\begin{array}{c}\text { Certified or reference } \\
\text { values }\end{array}$ & $\begin{array}{c}\text { Average percentage } \\
\text { of certified } \\
\text { or indicative values } \\
(\%)\end{array}$ \\
\hline \multirow{6}{*}{ NFRs } & PBT & nd & $n d^{a}$ & - \\
\hline & PBEB & 4.3 & $9.0^{a}$ & 48 \\
\hline & HBB & 4.8 & $4.5^{a}$ & 110 \\
\hline & EH-TBB & 90 & $26-54^{a-d}$ & $350-170$ \\
\hline & BEH-TEBP & 2300 & $500-1300$ a-d & $450-170$ \\
\hline & DBDPE & nd & $n d^{a-d}$ & - \\
\hline \multirow{11}{*}{ PBDEs } & BDE-17 & 12 & $12^{e}$ & 100 \\
\hline & BDE-28 & 47 & $47^{e}$ & 100 \\
\hline & BDE-47 & 500 & $500^{\circ}$ & 100 \\
\hline & BDE-85 & 53 & $44^{e}$ & 110 \\
\hline & BDE-99 & 1000 & $890^{e}$ & 110 \\
\hline & BDE-100 & 150 & $150^{e}$ & 99 \\
\hline & BDE-138 & 16 & $15^{e}$ & 100 \\
\hline & BDE-153 & 140 & $120^{e}$ & 110 \\
\hline & BDE-154 & 110 & $84^{e}$ & 120 \\
\hline & BDE-183 & 49 & $43^{e}$ & 110 \\
\hline & BDE-209 & 2100 & $2500^{e}$ & 94 \\
\hline
\end{tabular}

Brown et al., $2014(n=7)$

b Van den Eede et al., $2012(n=6)$

c Sahlström et al., $2012(n=5)$

d Ali et al., $2011(n=2)$

e SRM 2585, NIST 
Table S4: Recoveries (\%) of NFRs and PBDEs and surrogate standards in spiked wipe blanks $(n=3)$.

\begin{tabular}{|c|c|c|c|c|}
\hline Compound & Sample 1 & Sample 2 & Sample 3 & Mean \\
\hline TBP-AE & 95 & 110 & 94 & 98 \\
\hline PBBz & 100 & 110 & 98 & 100 \\
\hline PBT & 93 & 97 & 91 & 94 \\
\hline PBEB & 94 & 97 & 90 & 94 \\
\hline HBB & 110 & 110 & 110 & 110 \\
\hline EH-TBB & 96 & 98 & 95 & 97 \\
\hline BEH-TEBP & 91 & 89 & 100 & 94 \\
\hline s-DDC-CO & 110 & 99 & 110 & 110 \\
\hline a-DDC-CO & 110 & 100 & 110 & 110 \\
\hline OBTMPI & 45 & 48 & 65 & 53 \\
\hline DBDPE & 82 & 83 & 89 & 85 \\
\hline BDE-17 & 100 & 100 & 97 & 100 \\
\hline BDE-28 & 100 & 100 & 96 & 100 \\
\hline BDE-47 & 98 & 99 & 94 & 97 \\
\hline BDE-66 & 96 & 96 & 89 & 94 \\
\hline BDE-71 & 95 & 97 & 91 & 94 \\
\hline BDE-85 & 96 & 97 & 95 & 96 \\
\hline BDE-99 & 97 & 98 & 94 & 96 \\
\hline BDE-100 & 98 & 100 & 100 & 100 \\
\hline BDE-138 & 95 & 95 & 100 & 97 \\
\hline BDE-153 & 99 & 99 & 96 & 98 \\
\hline BDE-154 & 94 & 93 & 87 & 91 \\
\hline BDE-183 & 96 & 94 & 100 & 97 \\
\hline BDE-190 & 93 & 93 & 110 & 97 \\
\hline BDE-209 & 79 & 81 & 84 & 81 \\
\hline \multicolumn{5}{|c|}{ Surrogate Standards } \\
\hline mPBBz & 100 & 110 & 99 & 100 \\
\hline $\mathrm{mHBB}$ & 110 & 110 & 100 & 110 \\
\hline F-BDE-100 & 100 & 110 & 99 & 100 \\
\hline F-BDE-154 & 92 & 95 & 90 & 92 \\
\hline F-BDE-208 & 62 & 68 & 85 & 72 \\
\hline
\end{tabular}


Table S5: Spearman's rho correlation coffecients for NFRs and PBDEs in bedroom dust for compounds with $>70 \%$ DF. Only significant correlations are indicated.

\begin{tabular}{|c|c|c|c|c|c|c|c|c|c|c|c|c|c|}
\hline & \multicolumn{13}{|c|}{ PBDEs } \\
\hline BR Dust & 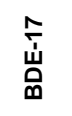 & 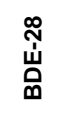 & $\begin{array}{l}\text { f } \\
\text { 山् } \\
\text { ه }\end{array}$ & $\begin{array}{l}\stackrel{g}{d} \\
\text { 㟔 } \\
\text { 品 }\end{array}$ & 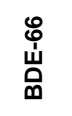 & $\begin{array}{l}\text { 吊 } \\
\text { 岀 } \\
\text { 口. }\end{array}$ & 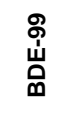 & $\begin{array}{l}\text { 옴 } \\
\dot{\dot{u}} \\
\text { }\end{array}$ & 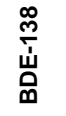 & 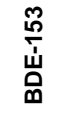 & 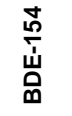 & $\begin{array}{l}\infty \\
\stackrel{\infty}{\dot{山}} \\
\stackrel{\infty}{0}\end{array}$ & 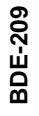 \\
\hline BDE_17 & 1 & & & & & & & & & & & & \\
\hline BDE_28 & .928 & 1 & & & & & & & & & & & \\
\hline BDE_47 & .866 & 909 & 1 & & & & & & & & & & \\
\hline BDE_49 & .751 & .733 & .716 & 1 & & & & & & & & & \\
\hline BDE_66 & .837 & .814 & .931 & .668 & 1 & & & & & & & & \\
\hline BDE_85 & .731 & .793 & .895 & 610 & .855 & 1 & & & & & & & \\
\hline BDE_99 & .786 & .848 & .960 & 661 & 908 & .950 & 1 & & & & & & \\
\hline BDE_100 & .739 & .777 & . 887 & .583 & .847 & .920 & 944 & 1 & & & & & \\
\hline BDE_138 & .676 & .700 & .852 & .554 & .845 & 931 & .932 & 918 & 1 & & & & \\
\hline BDE_153 & .691 & .676 & .783 & .607 & .808 & .842 & .855 & .833 & .881 & 1 & & & \\
\hline BDE_154 & .782 & .828 & .926 & .653 & .883 & 964 & .982 & 959 & .955 & .893 & 1 & . & \\
\hline BDE_183 & & & & & .355 & .432 & .343 & .383 & .504 & .424 & .415 & 1 & \\
\hline BDE_209 & & & & & & & & & & & & & 1 \\
\hline
\end{tabular}


Table S6: p-values from Wilcoxon signed rank tests for differences of NFRs and PBDEs between paired samples in the air and dust of bedrooms (BRs) and most used rooms (MURs) and paired hand palms versus backs (in blue). p-values for Mann-Whitney U tests for differences between hand-held versus non-hand-held e-devices (in red) with median concentrations in parentheses. Hand-held devices were cell phones, home phones, and tablets, while non-hand-held devices were TVs, stereos, and desktop computers. We tested the difference between median concentrations of hand-held vs non-handheld e-devices when laptops were categorized as either non-hand-held ${ }^{1}$ vs hand-held ${ }^{2}$.

\begin{tabular}{|c|c|c|c|c|c|}
\hline & \multicolumn{2}{|c|}{ BRs - MURs } & \multirow{2}{*}{$\begin{array}{c}\text { hands } \\
\begin{array}{c}\text { palms vs } \\
\text { backs }\end{array}\end{array}$} & \multicolumn{2}{|c|}{$\begin{array}{l}\text { Hand-held vs non-hand-held (median } \\
\text { concentrations in parentheses, } \mathrm{pg} / \mathrm{cm}^{2} \text { ) }\end{array}$} \\
\hline & air & dust & & $\begin{array}{l}\text { laptops taken as non- } \\
\text { hand-held }\end{array}$ & $\begin{array}{l}\text { laptops taken as } \\
\text { hand-held }\end{array}$ \\
\hline TBP-AE & 0.069 & 0.0010 & * & * & * \\
\hline PBBz & 0.012 & * & * & * & * \\
\hline PBT & 0.011 & 0.24 & 0.015 & $0.087(0.060 ; 0.14)$ & $0.40(0.07 ; 0.19)$ \\
\hline EH-TBB & 0.62 & 0.76 & 0.044 & $<0.001(22 ; 3.5)$ & $<0.001(19 . ; 1.9)$ \\
\hline BEH-TEBP & * & 0.80 & 0.075 & $0.0060(17 ; 4.9)$ & $<0.001(15 ; 3.5)$ \\
\hline s-DDC-CO & * & 0.84 & 0.13 & $0.0090(0.51 ; 0.60)$ & $0.10(0.53 ; 0.61)$ \\
\hline a-DDC-CO & * & 0.58 & 0.15 & $0.012(0.68 ; 0.77)$ & $0.090(0.71 ; 0.77)$ \\
\hline OBTMPI & * & * & * & $<0.001(4.4 ; 4.9)$ & $<0.001(4.3 ; 5.3)$ \\
\hline DBDPE & * & 0.18 & 0.0020 & $<0.001(12 ; 82)$ & $<0.001(13 ; 160)$ \\
\hline $\mathrm{BDE}-17$ & 0.91 & 0.47 & 0.16 & * & * \\
\hline BDE-28 & 0.99 & 0.29 & 0.33 & * & * \\
\hline BDE-47 & 0.73 & 0.10 & 0.25 & $0.0010(8.6 ; 2.9)$ & $<0.001(9.2 ; 1.8)$ \\
\hline BDE-49 & 0.82 & 0.88 & 0.12 & $0.030(0.31 ; 0.19)$ & $0.91(0.31 ; 0.16)$ \\
\hline BDE-66 & * & 0.030 & 0.017 & $0.0010(0.21 ; 0.17)$ & $0.18(0.22 ; 0.15)$ \\
\hline BDE-71 & * & 0.065 & * & * & * \\
\hline BDE-85 & * & 0.051 & 0.43 & $0.0010(0.24 ; 0.21)$ & $0.59(0.28 ; 0.17)$ \\
\hline BDE-99 & 0.27 & 0.10 & 0.29 & $0.0010(8.1 ; 2.5)$ & $<0.001(8.7 ; 1.4)$ \\
\hline BDE-100 & * & 0.22 & 0.88 & $0.0080(0.39 ; 0.25)$ & $0.79(0.43 ; 0.19)$ \\
\hline BDE-138 & * & 0.17 & 0.91 & * & * \\
\hline BDE-153 & * & 0.0070 & 0.12 & $<0.001(0.27 ; 0.30)$ & $<0.001(0.26 ; 0.33)$ \\
\hline BDE-154 & * & 0.061 & 0.26 & $<0.001(0.24 ; 0.20)$ & $0.13(0.26 ; 0.18)$ \\
\hline BDE-183 & * & 0.98 & * & $<0.001(0.16 ; 0.24)$ & $<0.001(0.16 ; 0.28)$ \\
\hline BDE-209 & * & 0.88 & 0.0020 & $<0.001(27 ; 79)$ & $0.0010(32 ; 99)$ \\
\hline
\end{tabular}

* Results not presented due to low detection frequencies in that sample matrix. 
Table S7. Spearman's rho correlation coefficients between each pair of variables (only significant correlations presented) for NFRs and PBDEs.

\begin{tabular}{|c|c|c|c|c|c|c|c|c|c|c|c|c|c|c|c|c|c|c|c|c|c|c|c|c|c|c|}
\hline & \multicolumn{10}{|c|}{ NFRs } & \multicolumn{15}{|c|}{ PBDEs } \\
\hline & & \multirow[t]{2}{*}{ 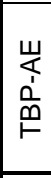 } & \multirow[t]{2}{*}{$\begin{array}{l}\tilde{M} \\
\underline{0} \\
\underline{\alpha}\end{array}$} & \multirow[t]{2}{*}{$\stackrel{5}{\circ}$} & \multirow[t]{2}{*}{ 恚 } & \multirow[t]{2}{*}{ 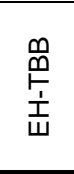 } & \multirow{2}{*}{ 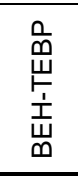 } & \multicolumn{2}{|c|}{$\begin{array}{l}\text { O } \\
\text { U. } \\
0\end{array}$} & \multirow[t]{2}{*}{ 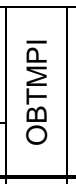 } & \multirow[t]{2}{*}{ 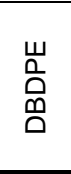 } & \multirow[t]{2}{*}{$\begin{array}{l}\hat{\overline{\dot{u}}} \\
\text { 品 }\end{array}$} & \multirow[t]{2}{*}{ 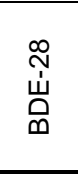 } & \multirow[t]{2}{*}{ 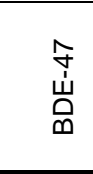 } & \multirow[t]{2}{*}{$\begin{array}{l}\stackrel{9}{+} \\
\stackrel{\leftrightarrow}{ }\end{array}$} & \multirow[t]{2}{*}{ 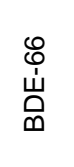 } & \multirow[t]{2}{*}{\begin{tabular}{|l|}
$\overline{1}$ \\
$\dot{山}$ \\
هิ
\end{tabular}} & \multirow[t]{2}{*}{ 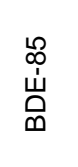 } & \multirow[t]{2}{*}{$\begin{array}{l}\text { ᄋ } \\
\dot{山} \\
\text { Ф̊ }\end{array}$} & \multirow{2}{*}{$\begin{array}{l}8 \\
\text { 响 } \\
\text { ه. }\end{array}$} & \multirow{2}{*}{ 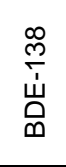 } & \multirow{2}{*}{ 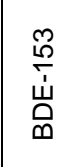 } & \multirow{2}{*}{ 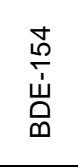 } & \multirow[t]{2}{*}{ 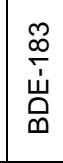 } & \multirow{2}{*}{$\begin{array}{l}\text { ○ } \\
\text { 岀 } \\
\text { ه̊ }\end{array}$} & \multirow{2}{*}{ 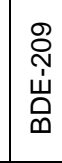 } \\
\hline & & & & & & & & is & $\dot{\sigma}$ & & & & & & & & & & & & & & & & & \\
\hline \multirow{2}{*}{$\begin{array}{l}\text { Hand palms } \\
\text { - Dust }\end{array}$} & BR Dust & & & $.39 \#$ & & $.69 \#$ & $.50 \#$ & & & & $.32^{*}$ & $.47 \#$ & $.56 \#$ & $.42 \#$ & & & & $.42 \#$ & $.35^{*}$ & $.54 \#$ & & $.32^{\star}$ & $.52 \#$ & & & \\
\hline & MUR Dust & & & $.39^{*}$ & & $.63 \#$ & $.64 \#$ & & & & & & $.57 \#$ & $.47^{\star}$ & & & & & $.43^{*}$ & $.43^{*}$ & & $.45^{\star}$ & $.42^{\star}$ & & & \\
\hline \multirow{7}{*}{$\begin{array}{l}\text { Hand palms } \\
\text { - Electronics }\end{array}$} & Cell Phones & & & & & $.48 \#$ & $.41^{\star}$ & & & & & & & $.48 \#$ & & & & $.48 \#$ & $.56 \#$ & & & & & & & \\
\hline & $\begin{array}{l}\text { Home } \\
\text { Phones } \\
\end{array}$ & & & & & $.68^{\star}$ & & & & & & & & & & & & $.66^{*}$ & & & & & .82\# & & & \\
\hline & Tablets & & & & & & & & & & & & & & & & & & & & & & & & & \\
\hline & Laptops & & & & & $.48 \#$ & $.39^{\star}$ & & & & & & & $.74 \#$ & $.41^{*}$ & & & $.59 \#$ & $.72 \#$ & & & $.37^{\star}$ & $.49 \#$ & & & \\
\hline & Desktops & & & & & & $.58^{\star}$ & & & & & & & & & & & & & & & & & & & \\
\hline & Televisions & & & & & & & & & & & & & $.34 \#$ & & & & & & & & & & & & \\
\hline & Stereos & & & & & & & $.82^{\star}$ & $.82^{\star}$ & & & & & & & & & & & & & & & & & \\
\hline Televisions - & BR Dust & & & & & $.25^{\star}$ & & & & & & & & & & & & & & & & & & & & \\
\hline & MUR Dust & & & $.47 \#$ & & & & & & & & & & & & & & & & & & & & & & \\
\hline
\end{tabular}

\# Correlation is significant at the 0.01 level (2-tailed).

* Correlation is significant at the 0.05 level (2-tailed). 


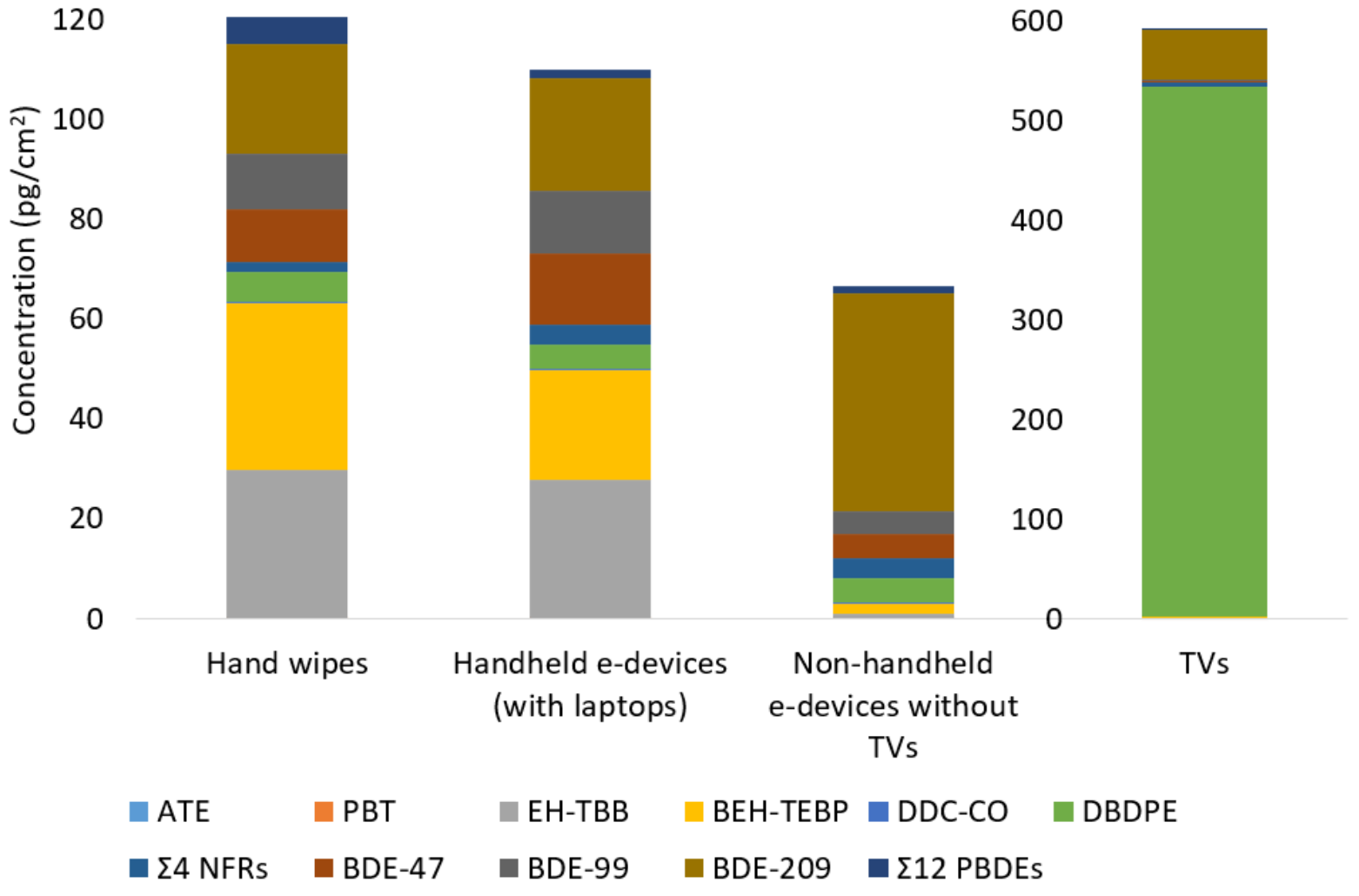

Fig. S1: Median concentrations of HFRs in hand (average of palm and back) and e-devices wipes. 


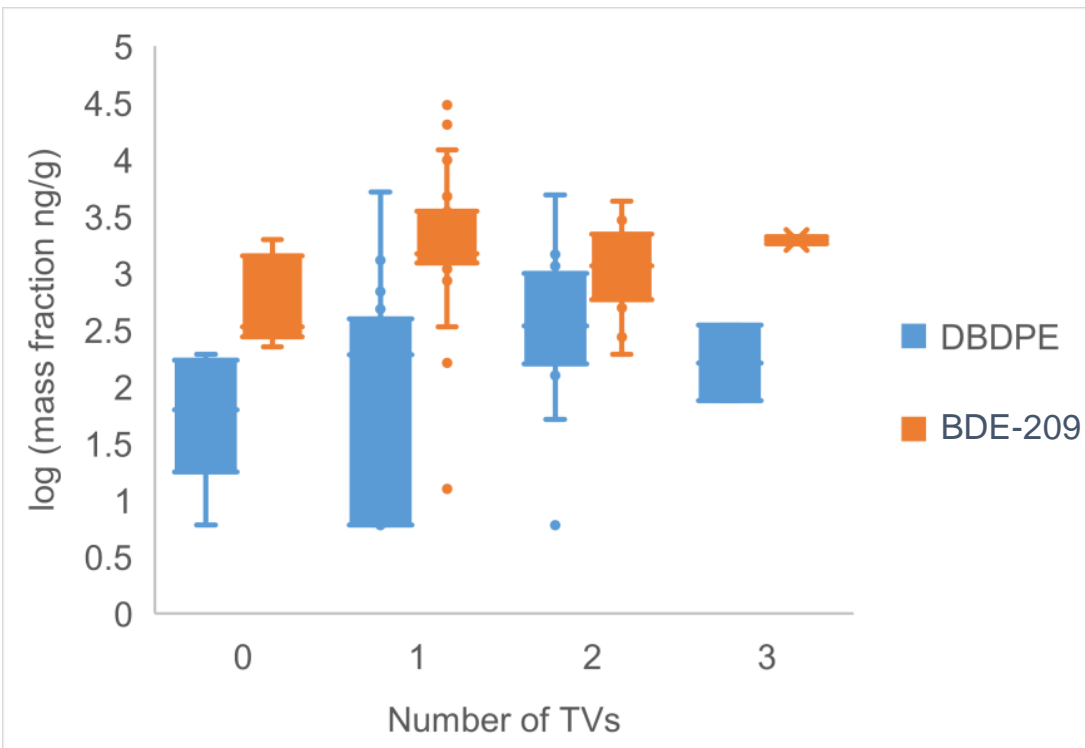

Fig. S2. DBDPE and BDE-209 mass fractions plotted against the number of TVs from the same homes. Boxes show first and third quartiles, whiskers define non-outlier range, and dots are outliers.

\section{References}

1. Harner T, Shoeib M. Measurements of octanol-air partition coefficients $\left(\mathrm{K}_{\mathrm{OA}}\right)$ for polybrominated diphenyl ethers (PBDEs): Predicting partitioning in the environment. J Chem Eng Data. 2002, 47:228-232. doi:10.1021/je010192t

2. Zhang X, Sühring R, Serodio D, Bonnell M, Sundin N, Diamond ML. Novel flame retardants: Estimating the physical-chemical properties and environmental fate of 94 halogenated and organophosphate PBDE replacements. Chemosphere. 2016, 144:2401-2407. doi:10.1016/j.chemosphere.2015.11.017 